\title{
Microscopic description of $\alpha$-decay as super-asymmetric fission
}

\author{
Mihail Mirea ${ }^{1,2, *}$ \\ ${ }^{1}$ Horia Hulubei National Institute for Physics and Nuclear Engineering, P.O. Box MG-6, 077125 \\ Bucharest-Magurele, Romania \\ ${ }^{2}$ Academy of Romanian Scientists, Splaiul Independentei 54, 050094 Bucharest, Romania
}

\begin{abstract}
The fine structure of $\alpha$-decay is treated with fission-like models. The single particle levels are calculated along a least action path connecting the ground state of the parent nucleus and the configuration of two spherical tangent nuclei. The probabilities to find different seniority-1 configurations are obtaining by solving the time-dependent pairing equations generalized by including the Landau-Zener effect and the Coriolis coupling. The theoretical results for the $\alpha$-decay of ${ }^{211} \mathrm{Po}$ and ${ }^{211} \mathrm{Bi}$ are compared with experimental data showing a good agreement.
\end{abstract}

\section{Introduction}

The $\alpha$ emission is usually explained as the formation of a cluster on the surface of the daughter nucleus followed by a penetrability of an external barrier at an energy close to the $Q$-value [1-15]. The probability to form such a combination is given by a square overlap of the convolution of the wave functions of the parent nucleus and that of the system at the touching configuration. The preformation amplitude depends on the initial and final states, without taking into account intermediate configurations. Recent advances for a more realistic treatment were obtained within the quartetting wave function approach [16-19].

In fission-like theories [20-22] of $\alpha$-decay, an extreme saturation of the nuclear matter is assumed [23]. Accordingly, the states of the nucleons should be strongly dependent on the boundaries of the many-body potential. When a preexisting $\alpha$ particle inside the parent nucleus is displaced towards the nuclear surface region, it perturbs all the microscopic states of the compound system. So, in a fission-like model, the rearrangement of the intrinsic states of the system during the formation of the combination consisting in both an $\alpha$ and a daughter nuclei should be taken into consideration.

In the formalism presented in the following, the rearrangement of the microscopic states during the emission of an $\alpha$-particle is investigated dynamically. At scission, we are left with two mean fields, one corresponding to the $\alpha$ cluster and another pertaining to the daughter nucleus. The probabilities to have the system in different final configurations should be determined.

For this purpose, a system of microscopic differential equations is deduced. These equations are managed by the variational principle [24]. They represent a generalization of the

\footnotetext{
*e-mail: mirea@ifin.nipne.ro
} 
time-dependent pairing equations by including two mechanisms: the Landau-Zener effect and the Coriolis coupling. Also, these equations provide information about the energy dissipated by the system. But in same time, we have an information concerning the distributions of intrinsic and collective angular momenta, and the momentary probabilities of different intrinsic configurations. The rearrangement in time of the nuclear orbitals during the disintegration process, starting from the initial compound nucleus and reaching the scission is investigated. As an application, the fine structure of the alpha-decay of an odd- $A$ mass nucleus is treated as the simplest modality to test the theory. The dynamics of the $\alpha$-decay process is revealed. From the least action principle, a superasymmetric fission path was obtained. The deformation energy is obtained in the framework of the macroscopic-microscopic model. The level scheme is calculated within the Woods-Saxon two center shell model. Consequently, the intensities of the transitions to the excited states of the daughter were evaluated theoretically. The experimental data were compared with the theoretical findings. A very good agreement was obtained. A mean value of the tunneling velocity of about $2 \times 10^{4} \mathrm{fm} / \mathrm{fs}$ was extracted.

\section{Microscopic equations of motion}

The coupled system of differential equations are obtained from the variational principle:

$$
\delta L=\delta\left\langle\varphi_{I M}\left|H+H_{R}-i \frac{\partial}{\partial t}+H^{\prime}-\lambda\right| N_{2} \hat{N}_{1}-N_{1} \hat{N}_{2}|| \varphi_{I M}\right\rangle
$$

where $H$ is the many-body Hamiltonian with pairing residual interaction, $H_{R}$ is the centrifugal contribution, $H^{\prime}$ is a term simulating the Landau-Zener effect [25], while $\hat{N}_{1}$ and $\hat{N}_{2}$ are particles numbers operators acting in the regions of spaces associated to the two nascent fragments. The condition obtained by means of the Lagrange multiplier $\lambda$ produces a dynamical projection of the numbers of particles on the two final fragments [26]. The trial wave functions $\left|\varphi_{I M}\right\rangle$ are considered as a superposition of seniority-1 Bogoliubov wave functions. After some algebras, the equations of motion follow [24, 27]:

$$
\begin{gathered}
-i \hbar \dot{c}_{\Omega, m}^{*}=c_{\Omega, m}^{*}\left\{2 \sum_{\left(\Omega^{\prime}, m^{\prime}\right) \neq(\Omega, m)}\left|v_{\Omega^{\prime}, m^{\prime}(\Omega, m)}\right|^{2}\left(\epsilon_{\Omega^{\prime}, m^{\prime}}-\lambda\right)+\left(\epsilon_{\Omega, m}-s_{k} \lambda N_{k}\right)\right. \\
\left.-G\left|\sum_{\left(\Omega^{\prime} m^{\prime}\right) \neq(\Omega, m)} u_{\Omega^{\prime}, m^{\prime}(\Omega, m)} v_{\Omega^{\prime}, m^{\prime}(\Omega, m)}\right|^{2}-G \sum_{\left(\Omega^{\prime}, m^{\prime}\right) \neq(\Omega, m)}\left|v_{\Omega^{\prime}, m^{\prime}(\Omega, m)}\right|^{4}\right\} \\
+\frac{\hbar^{2}}{2 J} c_{\Omega, m}^{*}\left[I(I+1)-\Omega^{2}\right]-\frac{\hbar^{2}}{2 J}\left\{\sum_{m^{\prime}} c_{\Omega+1, m^{\prime}}^{*}((I-\Omega)(I+\Omega+1))^{1 / 2}\right. \\
\times\left[u_{\Omega, m\left(\Omega+1, m^{\prime}\right)} u_{\Omega+1, m^{\prime}(\Omega, m)}+v_{\Omega, m\left(\Omega+1, m^{\prime}\right)}^{*} v_{\Omega+1, m^{\prime}(\Omega, m)}\right]\left\langle\Omega+1, m^{\prime}\left|j_{+}\right| \Omega, m\right\rangle T_{\Omega+1, m^{\prime}, \Omega, m} \\
+\sum_{m^{\prime}} c_{\Omega-1, m^{\prime}}^{*}((I+\Omega)(I-\Omega+1))^{1 / 2} \\
\left.\times\left[u_{\Omega, m\left(\Omega-1, m^{\prime}\right)} u_{\Omega-1, m^{\prime}(\Omega, m)}+v_{\Omega, m\left(\Omega-1, m^{\prime}\right)}^{*} v_{\Omega-1, m^{\prime}(\Omega, m)}\right]\left\langle\Omega-1, m^{\prime}\left|j_{-}\right| \Omega, m\right\rangle T_{\Omega-1, m^{\prime}, \Omega, m}\right\} \\
-i \hbar c_{\Omega, m}^{*} \sum_{\left(\Omega^{\prime}, m^{\prime}\right) \neq(\Omega, m)} \frac{1}{2}\left(v_{\Omega^{\prime}, m^{\prime}(\Omega, m)}^{*} \dot{v}_{\Omega^{\prime}, m^{\prime}(\Omega, m)}-\dot{v}_{\Omega^{\prime} m^{\prime}(\Omega, m)}^{*} v_{\Omega^{\prime}, m^{\prime}(\Omega, m)}\right) \\
+\sum_{m^{\prime} \neq m} h_{\Omega, m^{\prime}, m} c_{\Omega, m^{\prime}}^{*} T_{\Omega, m^{\prime}, \Omega, m}
\end{gathered}
$$

for the configuration mixing and

$$
\begin{aligned}
& -i \hbar \dot{v}_{\Omega^{\prime}, m^{\prime}(\Omega, m)}^{*}=2 v_{\Omega^{\prime}, m^{\prime}(\Omega, m)}^{*}\left(\epsilon_{\Omega^{\prime}, m^{\prime}}-\lambda\right)-G \sum_{\left(\Omega^{\prime \prime}, m^{\prime \prime}\right) \neq(\Omega, m)}\left\{u_{\Omega^{\prime \prime}, m^{\prime \prime}(\Omega, m)} v_{\Omega^{\prime \prime}, m^{\prime \prime}(\Omega, m)}^{*}\right. \\
& \left.\times\left(u_{\Omega^{\prime}, m^{\prime}(\Omega, m)}-\frac{v_{\Omega^{\prime}, m^{\prime}(\Omega, m)} v_{\Omega^{\prime}, m^{\prime}(\Omega, m)}^{*}}{2 u_{\Omega^{\prime}, m^{\prime}(\Omega, m)}}\right)-u_{\Omega^{\prime \prime}, m^{\prime \prime}(\Omega, m)} v_{\Omega^{\prime \prime}, m^{\prime \prime}(\Omega, m)} \frac{v_{\Omega^{\prime}, m^{\prime}(\Omega, m)}^{*} u_{\Omega^{\prime}, m^{\prime}(\Omega, m)}^{*}}{2 u_{\Omega^{\prime}, m^{\prime}(\Omega, m)}}\right\} \\
& -2 G v_{\Omega^{\prime}, m^{\prime}(\Omega, m)} v_{\Omega^{\prime}, m^{\prime}(\Omega, m)}^{*} v_{\Omega^{\prime}, m^{\prime}(\Omega, m)}^{*} .
\end{aligned}
$$

for the time-dependent pairing equations. In Eq. (2), $I$ is the total spin of the nuclear system, $\Omega$ is the projection of the intrinsic spin on the axial axis of symmetry, $m$ is a number that indexes the single-particle level in the pairing active levels space, $\lambda$ is a Lagrange multiplier, $s_{k}$ has values \pm 1 contributing to the dynamical projection of the number of particles $N_{k}$ of the 
nascent fragments, $G$ is the pairing strength, $J$ is the moment of inertia calculated with the cranking approximation, $T_{\gamma}$ are overlaps between Bogoliubov wave functions. The mixing between two seniority-1 configurations having the unpaired nucleons located in the states $(\Omega \pm 1, m)$ and $\left(\Omega, m^{\prime}\right)$ that differ by one unit in the projection of the intrinsic spin is produced by means of the matrix elements of the Coriolis coupling $\left\langle\Omega \mp 1, m^{\prime}\left|j_{\mp}\right| \Omega, m\right\rangle$. The mixing between two single particle levels having the same good quantum numbers $\Omega$ is realized by means of the Landau-Zener interaction strength $h_{\Omega, m, m^{\prime}}$. The parameter $c_{\Omega, m}$ is the amplitude of the seniority-1 wave function in which the single particle level $(\Omega, m)$ is blocked. The parameters $u_{\Omega, m\left(\Omega^{\prime}, m^{\prime}\right)}$ and $v_{\Omega, m\left(\Omega^{\prime}, m\right)}$ are the BCS vacancy and occupation amplitudes of the single particle level $(\Omega, m)$ in the seniority-1 configuration $\left(\Omega^{\prime}, m^{\prime}\right)$. The Rels. (3) are the well known time-dependent pairing equation, formally similar to the time-dependent Hartree-Fock equations $[28,29]$. By including the Landau-Zener promotion mechanism, these equations were able to explain microscopically the energy partition in low energy fission $[26,30]$ or the odd-even effect in cold fission [31,32].

\section{Results}

The fine structure of ${ }^{211} \mathrm{Po}$ and ${ }^{211} \mathrm{Bi} \alpha$-decays are reported, the daughter nuclei being ${ }^{207} \mathrm{~Pb}$ and ${ }^{207} \mathrm{Ta}$, respectively. A superasymmetric fission path between the ground state of the parent nucleus and the scission configuration is obtained according to the least action principle. In this context, the penetrability in the WKB approximation

$$
P=\exp \left\{-\frac{2}{\hbar} \int_{R_{1}}^{R_{2}} \sqrt{2 V(R) B(R)} d r\right\}
$$

is minimized. Two ingredients are needed to perform the minimization, the deformation energy $V(R)$ and the effective mass $B(R)$. The deformation energy is obtained in the framework of the macroscopic-microscopic model [33] extended for binary system with different charge densities while the inertia is computed within the cranking approach [34-36]. To avoid the singularities in the inertia during the passage of the avoided crossing regions, the matrix elements of the Hamiltonian between the state of the blocked level and the paired level states are neglected. Recent formulas for odd-mass inertia can be found in Refs. [36, 37], which differ slightly between them but both produce singularities. The surface term of liquid drop part of the deformation energy is obtained with the Yukawa plus exponential model. The shell and pairing effects are obtained with the Strutinsky prescriptions [34] based on the Woods-Saxon two-center shell model [25]. A smooth transition of the whole system between the one-body configuration and a two-body one is managed by our nuclear shape parametrization. Our nuclear shape parametrization is determined by five generalized coordinates: the distance between the centers of the nascent fragments denoted $R$ in the following, the curvature of the neck, the mass-asymmetry and the deformations of both fragments. A transition between the shell and pairing effects of the parent nucleus to those of both individual nuclei is realized in the scission region where the distance between the centers of the fragments is about $R \approx$ $9 \mathrm{fm}$, as already made for the $\alpha$-decay of ${ }^{296} \mathrm{Lv}$ in Ref. [38].

The barriers for the $\alpha$-decay of the two investigated parent nuclei are plotted in Fig. 1. A molecular minimum is produced for a distance between the centers of the fragments $R \approx 7 \mathrm{fm}$, which is due to the appearance of a strong shell effects produced by the reorganization of the single particle levels in a way compatible to the formation of the daughters, as explained previously in Refs. [20,38]. The daughters fail to reproduce the magic numbers by only one nucleon missing. A such molecular minimum was also noticed in Hartree-Fock treatments of $\alpha$-decay [16, 39]. The barriers of $\alpha$-decay resemble with the double humped fission barriers 


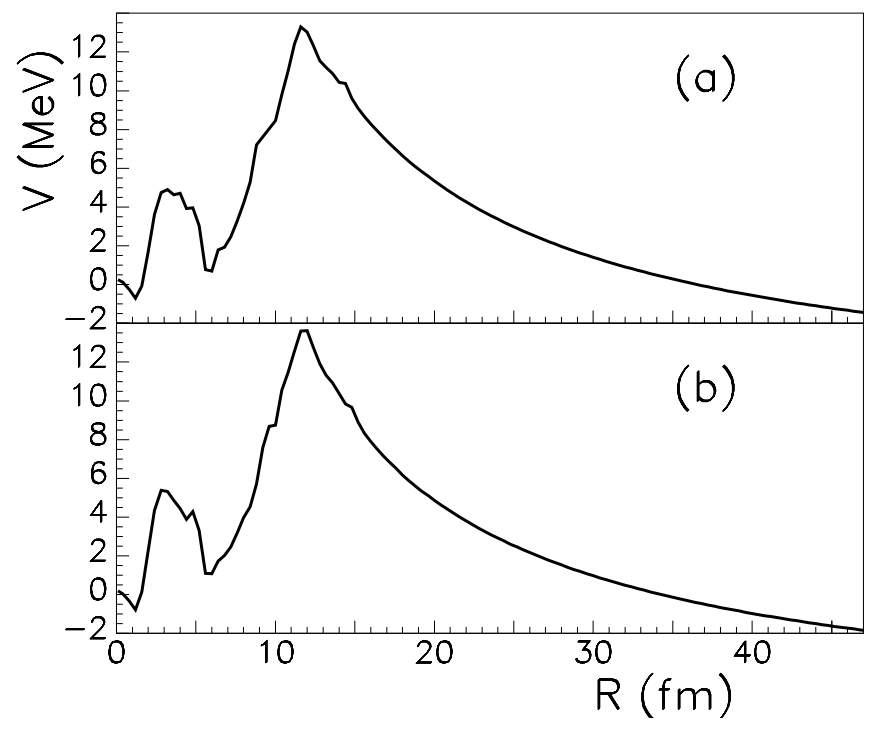

Figure 1. The $\alpha$-decay superasimmetric fission barriers are plotted with for ${ }^{211}$ Po in panel (a) and for ${ }^{211} \mathrm{Bi}$ in panel (b). The barriers are renormalized for an zero point kinetic energy of $0.5 \mathrm{MeV}$ in the ground state. Figure compiled from Refs. [24, 27].

Table 1. Comparison between experimental and theoretical transition probabilities $P_{x}$ on different final configurations $x=0,1,2$ of the daughter nucleus. The index $0,1,2$ mean the ground state, the first excited state and the second excited state, respectively. The orbitals of the daughter nucleus blocked by the unpaired nucleon for different values of $x$ are given in a separated row. The first column gives the parent nucleus involved in the $\alpha$-decay process. The following three columns addresses experimental transition probabilities (Exp) together with the final orbital for the lowest energy states. The last three columns are reserved for theoretical results (The). The experimental data are from Refs. [49, 50].

\begin{tabular}{lllllll}
\hline $\begin{array}{l}\text { Parent } \\
\text { nucleus }\end{array}$ & $\begin{array}{l}P_{0} \\
(\mathrm{Exp})\end{array}$ & $\begin{array}{l}P_{1} \\
(\mathrm{Exp})\end{array}$ & $\begin{array}{l}P_{2} \\
(\mathrm{Exp})\end{array}$ & $\begin{array}{l}P_{0} \\
(\mathrm{The})\end{array}$ & $\begin{array}{l}P_{1} \\
(\mathrm{The})\end{array}$ & $\begin{array}{l}P_{2} \\
(\mathrm{The})\end{array}$ \\
\hline${ }^{211} \mathrm{Po}$ & $3 p_{1 / 2}$ & $2 f_{5 / 2}$ & $3 p_{3 / 2}$ & $3 p_{1 / 2}$ & $2 f_{5 / 2}$ & $3 p_{3 / 2}$ \\
${ }^{211} \mathrm{Po}$ & $98.9 \%$ & $0.55 \%$ & $0.54 \%$ & $99.1 \%$ & $0.26 \%$ & $0.62 \%$ \\
\hline${ }^{211} \mathrm{Bi}$ & $3 s_{1 / 2}$ & $2 d_{3 / 2}$ & $1 h_{11 / 2}$ & $3 s_{1 / 2}$ & $2 d_{3 / 2}$ & $1 h_{11 / 2}$ \\
${ }^{211} \mathrm{Bi}$ & $83.77 \%$ & $16.23 \%$ & $<0.0018 \%$ & $87.9 \%$ & $12.1 \%$ & $6 \times 10^{-15}$ \\
\hline
\end{tabular}

as postulated by theories [40-43]. Therefore, it can be predicted that $\beta$-vibrational states $[44,45]$ are produced in the second potential well, explaining some anormal transition states observed experimentally [46, 47]

The microscopic equations of motion (2) and (3) are resolved along the superasymmetric fission path for the $\alpha$-decay of the two parent nuclei as detailed in Refs. [24, 27]. The Coriolis couplings and the single particle energies were determined within the two center WoodsSaxon wave functions. The initial states are $\left[\pi\left(h_{9 / 2}\right)^{2} v\left(g_{9 / 2}\right)^{1}\right]_{9 / 2}$ and $\left[\pi\left(h_{9 / 2}\right)^{1} v\left(g_{9 / 2}\right)^{2}\right]_{9 / 2}$ for ${ }^{211} \mathrm{Po}$ and ${ }^{211} \mathrm{Bi}$, respectively, the total spin $I$ being $9 / 2$. The momentum of inertia was computed in the framework of the cranking model. The Landau-Zener avoided crossings regions were identified in a consistent way. In a true avoided levels crossing region, as discussed in Ref. [48], the levels should exchange their characteristics. So, in each region of closest approach between two levels with the same good quantum numbers, an avoided levels crossing 
region was confirmed by the exchange of the intrinsic angular momenta at the same deformation. The magnitudes of the Landau-Zener interactions were extracted from the distances of closest approach between two adiabatic levels. Therefore, all the ingredients needed to solve the equations of motion are available. Only the internuclear velocity $\dot{R}$ is missing. The internuclear velocity is considered as a fitting parameter. For a initial configuration, the equations of motion provide all the possible final configurations and their probabilities of realization at scission. For each configuration characterized by an orbital angular momentum and a single particle excitation, I calculated the barrier penetrability. The products of configuration probabilities and penetrabilities are combined for each final orbital of the quasi-spherical daughter giving probabilities of transitions on different states, after a proper normalization. The best agreement between theory and experiment was obtained for an internuclear distance velocity of $\dot{R}=2 \times 10^{4} \mathrm{fm} / \mathrm{fs}$. A comparison between experimental data and theoretical results is given in table 1. At this velocity, the dissipated energy can be neglected [51]. Other microscopic models predict larger velocities [52].

\section{Conclusions}

The fine structure of $\alpha$-decay can be investigated microscopically with fission-like theories. A superasymmetric fission path compatible with the $\alpha$-decay process was obtained. The microscopic equations of motion, extended to include configuration mixing mechanisms, were resolved. The probabilities to obtain different configurations at scission were calculated by starting from the ground state of the parent nucleus. This probabilities offer the possibility to evaluate the fine structure characteristics for $\alpha$-decay. The theoretical results succeeded to reproduce well the experimental data.

This work was supported by the grant of Ministery of Research and Innovation, CNCS-UEFISCDI, project numbers PN-III-P4-ID-PCE-2016-0092, within PNCDI III.

\section{References}

[1] H.J. Mang, Z. Phys. 148, 582 (1957)

[2] H.J. Mang, Ann. Rev. Nucl. Sci. 14, 1 (1964)

[3] A. Sandulescu and M. Iosifescu, Nucl. Phys. 26, 209 (1961)

[4] D. Deng, Z. Ren, D. Ni, and Y Qian, J. Phys. G 42, 075106 (2015)

[5] D.S. Delion, Z.Z. Ren, A. Dumitrescu, and D.D. Ni, J. Phys. G 45, 053001 (2018)

[6] W.M. Seif and A. Adel, Phys. Rev. C 99, 044311 (2019)

[7] A. Adel and T. Alharbi, Nucl. Phys. A 975, 1 (2018)

[8] V. Dehghami, S.A. Alavi, and Kh. Benam, Mod. Phys. Lett. A 33, 1850080 (2018)

[9] G.M. Carmel Viola Bai and R. Nithya Agnes, Pramana 93, 39 (2019)

[10] A.O. Silisteanu, C.I. Anghel, and I. Silisteanu, Rom. J. Phys. 63, 302 (2018)

[11] H.M. Devajara, Y.K. Gambir, A. Bhagwat, M. Gupta, S. Heinz and G. Munzenberg, Rom. J. Phys. 63, 304 (2018)

[12] A. Soylu, Chin. Phys. C 43, 074102 (2019)

[13] M. Ismail and A. Adel, J. Phys. G 44, 125106 (2017)

[14] D.S. Delion, Phys. Rev. C 80, 024310 (2009)

[15] K.P. Santhosh, J.G. Joseph and B. Priyanka, Nucl. Phys. A 877, 1 (2012)

[16] C. Xu, G. Röpke, P. Schuck, Z. Ren, Y. Funaki, H. Horiuchi, A. Tohsaki, T. Yamada, and B. Zhou, Phys. Rev. C 95, 061306(R) (2017) 
[17] G. Röpke, P. Schuck, Y. Funaki, H. Horiuchi, Z. Ren, A. Tohsaki, C. Xu, T.Yamada, and B. Zhou, Phys. Rev. C 90, 034304 (2014)

[18] G. Röpke, P. Schuck, C. Xu, Z. Ren, M. Lyu, B. Zhou, Y. Funaki, H. Horiuchi, A. Tohsaki, and T. Yamada, J. Low Temp. Phys. 189, 383 (2017)

[19] D.S. Delion, A. Dumitrescu, and V.V. Baran, Phys. Rev. C 97, 064303 (2018)

[20] M. Mirea, Eur. Phys. J. A 51, 36 (2015)

[21] M. Mirea, R. Budaca, and A. Sandulescu, Ann. Phys. 380, 154 (2017)

[22] A. Sandulescu and M. Mirea, Eur. Phys. J. A 50, 110 (2014)

[23] D.L. Hill and J.A. Wheeler, Phys. Rev. 89, 1102 (1953)

[24] M. Mirea, Phys. Rev. C 96, 064607 (2017)

[25] M. Mirea, Phys. Rev. C 78, 044618 (2008)

[26] M. Mirea, Phys. Rev. C 89, 034623 (2014)

[27] M. Mirea, EPL 124, 12001 (2018)

[28] S.E. Koonin and J.R. Nix, Phys. Rev. C 13, 209 (1976)

[29] J. Blocki and H. Flocard, Nucl. Phys. A 273, 45 (1976)

[30] M. Mirea, Phys. Lett. B 717, 252 (2012)

[31] M. Mirea, Phys. Lett. B 680, 316 (2009)

[32] M. Mirea, Phys. Rev. C 83, 054608 (2011)

[33] P. Moller, J.R. Nix, W.D. Myers, and W.J. Swiatecki, At. Data. Nucl. Data. Tables 59 185 (1995)

[34] M. Brack, J. Damgaard, A. Jensen, H. Pauli, V. Strutinsky, and W. Wong, Rev. Mod. Phys. 44, 320 (1972)

[35] M. Mirea, J. Phys. G 43, 105103 (2016)

[36] M. Mirea, Phys. Rev. C 100, 014607 (2019)

[37] W. Brodzinski, M. Kowal, J. Skalski, and P. Jachimowicz, Acta Phys. Pol. B 49, 621 (2018)

[38] A. Sandulescu, M. Mirea, and D.S. Delion, EPL 101, 62001 (2013)

[39] G.G. Adamian, N.V. Antonenko, H. Lenske, S.V. Tolokonnikov, and E.E. Saperstein, Phys. Rev. C 94, 054309 (2016)

[40] K.-H. Schmidt and B. Jurado, Rep. Prog. Phys. 81, 106301 (2018)

[41] M. Mirea, Int. J. Mod. Phys. E 27, 1850076 (2018)

[42] Zhiming Wang, Zhong Zhu, CL. Zhong, and TS. Fan, Nucl. Phys. A 989, 81 (2019)

[43] Bing-Nan Lu, E.G. Zhao, and S.G. Zhou, Phys. Rev. C 85, 011301 (2012)

[44] S. Bjornholm and J.E. Lynn, Rev. Mod. Phys. 52, 725 (1980)

[45] M. Mirea, Rom. J. Phys. 64, 305 (2019)

[46] A. Astier, P. Petkov, M.-G. Porquet, D.S. Delion, and P. Schuck, Eur. Phys. J. A 46, 165 (2010)

[47] D.S. Delion and R.J. Liotta, Phys. Rev. C 87, 041302(R) (2013)

[48] C. Zener, Proc. R. Soc. A 137, 696 (1932)

[49] L.J. Jardine, Phys. Rev. C 11, 1385 (1975)

[50] F.G. Kondev and S. Lalkovski, Nucl. Data Sheets 112, 707 (2011)

[51] M. Mirea and A. Sandulescu, Rom. Rep. Phys. 70, 201 (2018)

[52] O. Serot, N. Carjan, and D. Strottman, Nucl. Phys. A 569, 562 (1994) 